\title{
Application of $p$-regularity theory to the Duffing equation
}

\author{
Beata Medak ${ }^{1 *+}$ (D) and Alexey A Tret'yakov 1,2,3†
}

\author{
"Correspondence: \\ bmedak@uph.edu.pl \\ Siedlce University of Natural \\ Sciences and Humanities, 3-go Maja \\ 54, Siedlce, 08-110, Poland \\ Full list of author information is \\ available at the end of the article \\ ${ }^{\dagger}$ Equal contributors
}

\begin{abstract}
The paper studies a solution existence problem of the nonlinear Duffing equation of the form

$$
F(x, \mu, \beta)=x^{\prime \prime}+x+\mu x^{3}-\beta \sin t=0, \quad \beta>0, \mu \neq 0
$$

where $F: \mathcal{C}^{2}[0,2 \pi] \times \mathbb{R} \times \mathbb{R} \rightarrow \mathcal{C}[0,2 \pi]$ and $x(0)=x(2 \pi)=0$ using the $p$-regularity theory.

MSC: $47 \mathrm{~J} 05 ; 34 \mathrm{~B} 15 ; 34 \mathrm{~B} 16$

Keywords: $p$-regularity; $p$-factor operator; Duffing equation; nonlinear boundary value problems
\end{abstract}

\section{Introduction}

This paper is a continuation of the work by Grzegorczyk et al. [1]. We study the structure of solutions to the Duffing oscillation equation of the form

$$
F(x, \mu, \beta)=x^{\prime \prime}+x+\mu x^{3}-\beta \sin t=0, \quad \beta>0, \mu \neq 0,
$$

where $F: \mathcal{C}^{2}[0,2 \pi] \times \mathbb{R} \times \mathbb{R} \rightarrow \mathcal{C}[0,2 \pi]$ and $x(0)=x(2 \pi)=0$ applying some methods of $p$-regularity theory [2-5].

We consider a special case of the general Duffing equation given by

$$
x^{\prime \prime}+\delta x^{\prime}+\alpha x+\mu x^{3}=\beta \cos \omega t
$$

where the unknown function $x=x(t)$ is the displacement at time $t, x^{\prime}$ is the first derivative of $x$ with respect to time, i.e., velocity, and $x^{\prime \prime}$ is the second time-derivative of $x$, i.e., acceleration. The numbers $\delta, \alpha, \beta, \gamma$ and $\omega$ are given constants.

In general, the Duffing equation does not admit an exact symbolic solution. However, there are some approximate methods, i.e., expansion in a Fourier series, the Frobenius method, numeric methods such as Euler's and Runge-Kutta methods, the homotopy analysis method $[6,7]$. In this paper we present a new approach, the so-called $p$-regularity method, and show that this approximate method works well and is effective.

The Duffing equation is a degenerate problem since there exists $x^{*}$ such that it is a solution, but $\operatorname{Im} F^{\prime}\left(x^{*}\right) \neq Y$. The construction of $p$-regularity gives possibilities for solving

(c) The Author(s) 2017. This article is distributed under the terms of the Creative Commons Attribution 4.0 International License (http://creativecommons.org/licenses/by/4.0/), which permits unrestricted use, distribution, and reproduction in any medium, provided you give appropriate credit to the original author(s) and the source, provide a link to the Creative Commons license, and indicate if changes were made. 
such nontrivial problems. This paper shows how to determine the asymptotic solutions in the case of degeneracy of order higher than two. In the work by Grzegorczyk et al. [1], the authors studied the 2-regularity case. Now we examine 4-regularity.

\section{The main results of $p$-regularity theory}

The apparatus of $p$-regularity is a new tool for studying nonlinear problems. In this section, we present some definitions, notations and theorems of $p$-regularity theory to be used in what follows (see $[1-5,8,9]$ ).

This paper deals with the nonlinear problem

$$
F(x)=0,
$$

where $F$ is a sufficiently smooth mapping between Banach spaces $X$ and $Y$. Apply $p$ regularity theory to this equation.

Assume that $x^{*} \in U \subseteq X, U$ is a neighborhood of the element $x^{*}$. Suppose that there is singularity at the point $x^{*}$. Assume that the space $Y$ can be decomposed into a direct sum of subspaces

$$
Y=Y_{1} \oplus \cdots \oplus Y_{p}
$$

where $Y_{1}=\operatorname{cl}\left(\operatorname{Im} F^{\prime}\left(x^{*}\right)\right)$ and $Z_{1}=Y$. As $Z_{2}$ we use the closed complement of $Y_{1}$ in $Y$. Let $P_{Z_{2}}: Y \rightarrow Z_{2}$ be the projector onto $Z_{2}$ along $Y_{1}$. By $Y_{2}$ we denote the closure of a linear span of the image of the quadratic mapping $P_{Z_{2}} F^{\prime \prime}\left(x^{*}\right)[\cdot]^{2}$. Then, inductively,

$$
Y_{i}=\operatorname{cl}\left(\operatorname{span} \operatorname{Im} P_{Z_{i}} F^{(i)}\left(x^{*}\right)[\cdot]^{i}\right) \subseteq Z_{i}, \quad i=2, \ldots, p-1,
$$

where $Z_{i}$ is a closed complement of $Y_{1} \oplus \cdots \oplus Y_{i-1}, i=2, \ldots, p$, with respect to $Y$, and $P_{Z_{i}}$ : $Y \rightarrow Z_{i}$ is a projector onto $Z_{i}$ along $Y_{1} \oplus \cdots \oplus Y_{i-1}, i=2, \ldots, p$, with respect to $Y$. Finally, $Y_{p}=Z_{p}$. The order $p$ is the minimal number (if it exists) for which the decomposition (4) holds. Define the mapping

$$
f_{i}: U \rightarrow Y_{i}, \quad f_{i}(x)=\Pi_{i} F(x), \quad i=1, \ldots, p,
$$

where $\Pi_{i}: Y \rightarrow Y_{i}$ is the projector onto $Y_{i}$ along $Y_{1} \oplus \cdots \oplus Y_{i-1} \oplus Y_{i+1} \oplus \cdots \oplus Y_{p}$. Then the mapping $F$ can be represented as

$$
F(x)=f_{1}(x)+\cdots+f_{p}(x) .
$$

Definition 1 The linear operator mapping $X$ to $Y$

$$
\Psi_{p}(h)=\Psi_{p}\left(x^{*}, h\right)=f_{1}^{\prime}\left(x^{*}\right)+f_{2}^{\prime \prime}\left(x^{*}\right)[h]+\cdots+f_{p}^{(p)}\left(x^{*}\right)[h]^{p-1}
$$

such that

$$
\Psi_{p}(h) x=\Psi_{p}\left(x^{*}, h\right) x=f_{1}^{\prime}\left(x^{*}\right) x+f_{2}^{\prime \prime}\left(x^{*}\right)[h] x+\cdots+f_{p}^{(p)}\left(x^{*}\right)[h]^{p-1} x, \quad x \in X,
$$

is called $p$-factor operator. 
Definition 2 A mapping $F$ is called $p$-regular at $x^{*}$ along $h(p>1)$ if $\operatorname{Im} \Psi_{p}(h)=Y$ (i.e., the operator $\Psi_{p}(h)$ is surjective).

Definition 3 The $p$-kernel of the operator $\Psi_{p}(h)$ is defined as a set

$$
\begin{aligned}
H_{p}\left(x^{*}\right) & =\operatorname{Ker}^{p} \Psi_{p}(h)=\left\{h \in X: \Psi_{p}(h)[h]=0\right\} \\
& =\left\{h \in X: f_{1}^{\prime}\left(x^{*}\right)[h]+f_{2}^{\prime \prime}\left(x^{*}\right)[h]^{2}+\cdots+f_{p}^{(p)}\left(x^{*}\right)[h]^{p}=0\right\} .
\end{aligned}
$$

Note that

$$
\operatorname{Ker}^{p} \Psi_{p}(h)=\left\{\bigcap_{i=1}^{p} \operatorname{Ker}^{i} f_{i}^{(i)}\left(x^{*}\right)\right\} .
$$

Definition 4 A mapping $F$ is called $p$-regular at $x^{*}(p>1)$ if either it is $p$-regular along every $h \in H_{p}\left(x^{*}\right) \backslash\{0\}$ or $H_{p}\left(x^{*}\right)=\{0\}$.

Define the solution set for the mapping $F$ as the set

$$
M=M\left(x^{*}\right)=\left\{x \in X: F(x)=F\left(x^{*}\right)=0\right\},
$$

and let $T_{x^{*}} M$ denote the tangent cone to the set $M$ at the point $x^{*}$, i.e.,

$$
T_{x^{*}} M=\left\{h \in X: x^{*}+\varepsilon h+r(\varepsilon) \in M,\|r(\varepsilon)\|=o(\varepsilon), \varepsilon \in[0, \delta], \delta>0\right\} .
$$

The following theorem describes the tangent cone to the solutions sets of equation (3) in the $p$-regular case.

Theorem 5 (Generalized Lyusternik theorem, [2]) Let $X$ and $Y$ be the Banach spaces, and let the mapping $F \in C^{p}(X, Y)$ be p-regular at $x^{*} \in M$. Then

$$
T_{x^{*}} M=H_{p}\left(x^{*}\right) .
$$

Let us explain that here (for Banach spaces $X$ and $Y) F \in C^{p}(X, Y)$ means that $F: X \rightarrow Y$ is $p$ times continuously Frechét differentiable.

\section{Solutions to the Duffing equation}

Consider the oscillation Duffing equation

$$
F(x, \mu, \beta)=x^{\prime \prime}+x+\mu x^{3}-\beta \sin t=0, \quad \beta>0, \mu \neq 0,
$$

where $F: \mathcal{C}^{2}[0,2 \pi] \times \mathbb{R} \times \mathbb{R} \rightarrow \mathcal{C}[0,2 \pi]$ and $x(0)=x(2 \pi)=0$.

Note that for $\mu=0, \beta>0$, solutions of the above equation do not exist, but for $\beta=0$, there exists zero solution. We are interested in nontrivial solutions of this equation of course. The following theorem holds. 
Theorem 6 For sufficiently small $\beta>0$, the Duffing equation (1) has nontrivial solution tangent to elements from the 4-kernel of the form

$$
\begin{aligned}
& x(\beta, t)=\frac{\beta^{\frac{1}{4}}}{\bar{\beta}^{\frac{1}{4}}}\left(\sqrt[3]{\frac{4 \bar{\beta}}{3 \bar{\mu}}} \sin t\right)+o\left(\beta^{\frac{1}{4}}\right), \\
& \mu(\beta)=\frac{\beta^{\frac{1}{4}}}{\bar{\beta}^{\frac{1}{4}}} \bar{\mu}+o\left(\beta^{\frac{1}{4}}\right),
\end{aligned}
$$

where $\bar{\mu}, \bar{\beta}$ are fixed numbers for which the following conditions hold: $3 \sqrt[3]{\frac{4 \bar{\beta}}{3 \mu}}+|\bar{\mu}|+\left|\bar{\beta}^{\frac{1}{4}}\right|=1$, $\bar{\mu}=\bar{\mu}\left(\bar{\beta}^{\frac{1}{4}}\right), \bar{\beta}=\bar{\alpha}^{4}$ and $0<\bar{\beta}^{\frac{1}{4}}<1$.

Proof In order to apply the theory of $p$-regularity, let us put $\beta^{\frac{1}{4}}=\alpha$ and go to an equivalent equation of the form

$$
\bar{F}(x, \mu, \alpha)=x^{\prime \prime}+x+\mu x^{3}-\alpha^{4} \sin t=0 .
$$

Exactly now we show that the mapping $\bar{F}$ is 4 -regular, and we will describe solutions. Of course, $x^{*}=(0,0,0)$ ais the trivial solution of the above equation. We have

$$
\begin{aligned}
\bar{F}^{\prime}(x, \mu, \alpha) & =\left(\bar{F}_{x}^{\prime}(x, \mu, \alpha), \bar{F}_{\mu}^{\prime}(x, \mu, \alpha), \bar{F}_{\alpha}^{\prime}(x, \mu, \alpha)\right) \\
& =\left(\frac{d^{2}}{d t^{2}}+1+3 \mu x^{2}, x^{3},-4 \alpha^{3} \sin t\right)
\end{aligned}
$$

and

$$
\bar{F}^{\prime}(0,0,0)=\left(\bar{F}_{x}^{\prime}(0,0,0), \bar{F}_{\mu}^{\prime}(0,0,0), \bar{F}_{\alpha}^{\prime}(0,0,0)\right)=\left(\frac{d^{2}}{d t^{2}}+1,0,0\right) .
$$

Note that $\operatorname{Ker} \bar{F}_{x}^{\prime}(0,0,0)=\left\{x \in \mathcal{C}^{2}[0,2 \pi]: \frac{d^{2} x}{d t^{2}}+x=0\right\}$. The general solutions of the equation $x^{\prime \prime}+x=0$ are $x(t)=c_{1} \cos t+c_{2} \sin t$. Taking into account the boundary conditions, we obtain $c_{1}=0, x(t)=c_{2} \sin t$ and $\operatorname{Ker} \bar{F}_{x}^{\prime}(0,0,0)=\operatorname{span}\{\sin t\}$.

The image of the operator $\bar{F}_{x}^{\prime}(0,0,0)$ is defined as follows:

$$
\begin{aligned}
\operatorname{Im} \bar{F}_{x}^{\prime}(0,0,0) & =\left\{y \in \mathcal{C}[0,2 \pi]: \exists x \in \mathcal{C}^{2}[0,2 \pi] \bar{F}_{x}^{\prime}(0,0,0) x=y, x(0)=x(2 \pi)=0\right\} \\
& =\left\{y \in \mathcal{C}[0,2 \pi]: \exists x \in \mathcal{C}^{2}[0,2 \pi] x^{\prime \prime}+x=y, x(0)=x(2 \pi)=0\right\} .
\end{aligned}
$$

The general solution of the equation $x^{\prime \prime}+x=y$ has the form

$$
x(t)=c_{1} \cos t+c_{2} \sin t-\cos t \int_{0}^{t} y(\tau) \sin \tau d \tau+\sin t \int_{0}^{t} y(\tau) \cos \tau d \tau .
$$

In view of the boundary condition, we obtain $c_{1}=0 \mathrm{i} \int_{0}^{2 \pi} y(\tau) \sin \tau d \tau=0$, then

$$
\begin{aligned}
\operatorname{Im} \bar{F}_{x}^{\prime}(0,0,0) & =\left\{y \in \mathcal{C}[0,2 \pi]: \int_{0}^{2 \pi} y(\tau) \sin \tau d \tau=0\right\} \\
& =\left\{y \in \mathcal{C}[0,2 \pi]:\langle y, \sin t\rangle=0, \sin t \in \operatorname{Ker} \bar{F}_{x}^{\prime}(0,0,0)\right\} .
\end{aligned}
$$


One can easily show that the boundary value problem $x^{\prime \prime}+x=\sin t, x(0)=x(2 \pi)=0$ does not have a solution. This implies that the operator $\bar{F}_{x}^{\prime}(0,0,0)$ is not surjective and $\operatorname{Im} \bar{F}_{x}^{\prime}(0,0,0) \neq \mathcal{C}[0,2 \pi]=Y$. Then $Y=Y_{1} \oplus Z_{2}$, where $Y_{1}=\operatorname{Im} \bar{F}_{x}^{\prime}(0,0,0), Z_{2}=Y_{1}^{\perp}$.

The projector $P_{Z_{2}}: Y \rightarrow Z_{2}$ can be described as

$$
P_{Z_{2}} y=\frac{1}{\pi} \sin t\langle y, \sin t\rangle=\frac{1}{\pi} \sin t \int_{0}^{2 \pi} y(\tau) \sin \tau d \tau, \quad y \in Y .
$$

This implies that

$$
\begin{aligned}
Y_{2} & =\operatorname{span}\left(\operatorname{Im} P_{Z_{2}} \bar{F}^{\prime \prime}(0,0,0)[\cdot]^{2}\right) \\
& =\operatorname{span}\left\{y(t) \in Y: y(t)=\frac{1}{\pi} \sin t \int_{0}^{2 \pi} \bar{F}^{\prime \prime}(0,0,0)[z(\tau)]^{2} \sin \tau d \tau, z(\tau) \in \mathcal{C}^{2}[0,2 \pi]\right\} \\
& \subseteq Z_{2} .
\end{aligned}
$$

Let us evaluate the second derivative of the mapping $\bar{F}$

$$
\bar{F}^{\prime \prime}(x, \mu, \alpha)=\left(\left(6 \mu x, 3 x^{2}, 0\right),\left(3 x^{2}, 0,0\right),\left(0,0,-12 \alpha^{2} \sin t\right)\right) .
$$

From this we obtain

$$
\bar{F}^{\prime \prime}(0,0,0)=0
$$

and $Y_{2}=\{0\}, Z_{3}=\left(Y_{1} \oplus\{0\}\right)^{\perp}$. We continue

$$
\begin{aligned}
P_{Z_{3}} y & =\frac{1}{\pi} \sin t\langle y, \sin t\rangle=\frac{1}{\pi} \sin t \int_{0}^{2 \pi} y(\tau) \sin \tau d \tau, \quad y \in Y, \\
Y_{3}= & \operatorname{span}\left(\operatorname{Im} P_{Z_{3}} \bar{F}^{\prime \prime \prime}(0,0,0)[\cdot]^{3}\right) \\
= & \operatorname{span}\left\{y(t) \in Y: y(t)=\frac{1}{\pi} \sin t \int_{0}^{2 \pi} \bar{F}^{\prime \prime \prime}(0,0,0)[z(\tau)]^{3} \sin \tau d \tau, z(\tau) \in \mathcal{C}^{2}[0,2 \pi]\right\} \\
& \subseteq Z_{3} .
\end{aligned}
$$

Let us evaluate the third derivative of the mapping $\bar{F}$ :

$$
\begin{aligned}
\bar{F}^{\prime \prime \prime}(x, \mu, \alpha)= & (((6 \mu, 6 x, 0)(6 x, 0,0),(0,0,0)),((6 x, 0,0),(0,0,0),(0,0,0)), \\
& ((0,0,0),(0,0,0),(0,0,-24 \alpha \sin t))) .
\end{aligned}
$$

From this

$$
\bar{F}^{\prime \prime \prime}(0,0,0)=0
$$

and $Y_{3}=\{0\}$ i $Y=Y_{1} \oplus\{0\} \oplus\{0\} \oplus Z_{4}, Z_{4}=\left(Y_{1} \oplus\{0\} \oplus\{0\}\right)^{\perp}$. Next we have

$$
P_{Z_{4}} y=\frac{1}{\pi} \sin t\langle y, \sin t\rangle=\frac{1}{\pi} \sin t \int_{0}^{2 \pi} y(\tau) \sin \tau d \tau, \quad y \in Y
$$


and

$$
\begin{aligned}
Y_{4} & =\operatorname{span}\left(\operatorname{Im} P_{Z_{4}} \bar{F}^{(4)}(0,0,0)[\cdot]^{4}\right) \\
& =\operatorname{span}\left\{y(t) \in Y: y(t)=\frac{1}{\pi} \sin t \int_{0}^{2 \pi} \bar{F}^{(4)}(0,0,0)[z(\tau)]^{4} \sin \tau d \tau, z(\tau) \in \mathcal{C}^{2}[0,2 \pi]\right\} .
\end{aligned}
$$

Let us evaluate the fourth derivative:

$$
\begin{aligned}
& \bar{F}^{(4)}(x, \mu, \alpha) \\
&=(((0,6,0),(6,0,0),(0,0,0)),((6,0,0),(0,0,0),(0,0,0)), \\
&((0,0,0),(0,0,0),(0,0,0))),(((6,0,0),(0,0,0),(0,0,0)), \\
&((0,0,0),(0,0,0),(0,0,0)),((0,0,0),(0,0,0),(0,0,0))), \\
&(((0,0,0),(0,0,0),(0,0,0)),((0,0,0),(0,0,0),(0,0,0)), \\
&((0,0,0),(0,0,0),(0,0,-24 \sin t)))) \\
&= \bar{F}^{(4)}(0,0,0) .
\end{aligned}
$$

Note that $\bar{F}^{(5)}(x, \mu, \alpha)=0, Z_{4}=Y_{4}$ and $P_{Z_{4}} y=\Pi_{Y_{4}} y$. Therefore we will examine 4regularity.

Now let us take $z(\tau)=\left(h_{x}, h_{\mu}, h_{\alpha}\right)$. For such a defined vector $z(\tau)$, the following relation holds:

$$
\begin{aligned}
& \bar{F}^{(4)}(0,0,0)[z(\tau)]^{3}=\left(18 h_{x}^{2} h_{\mu}, 6 h_{x}^{3},-24 \sin \tau h_{\alpha}^{3}\right) \\
& \bar{F}^{(4)}(0,0,0)[z(\tau)]^{4}=24\left(h_{x}^{3} h_{\mu}-\sin \tau h_{\alpha}^{4}\right)
\end{aligned}
$$

and we can describe the subspace

$$
\begin{aligned}
Y_{4} & =\operatorname{span}\left\{y(t) \in Y: y(t)=\frac{24}{\pi} \sin t \int_{0}^{2 \pi}\left(h_{x}^{3} h_{\mu}-\sin \tau h_{\alpha}^{4}\right) \sin \tau d \tau\right\} \\
& =\operatorname{span}\{\sin t\}=\operatorname{Ker} \bar{F}_{x}^{\prime}(0,0,0),
\end{aligned}
$$

4-factor operator

$$
\begin{aligned}
& \forall u=\left(h_{u}, h_{\lambda}, h_{\beta}\right) \in C^{2}[0,2 \pi] \times \mathbb{R} \times \mathbb{R} \\
& \begin{aligned}
\Psi_{4}(h)[u] & =\Psi_{4}\left((0,0,0),\left(h_{x}, h_{\mu}, h_{\alpha}\right)\right)\left(h_{u}, h_{\lambda}, h_{\beta}\right) \\
& =\frac{d^{2} h_{u}}{d t^{2}}+h_{u}+\frac{1}{\pi} \sin t \int_{0}^{2 \pi}\left(18 h_{x}^{2} h_{\mu} h_{u}+6 h_{x}^{3} h_{\lambda}-24 \sin \tau h_{\alpha}^{3} h_{\beta}\right) \sin \tau d \tau
\end{aligned}
\end{aligned}
$$

and the 4-kernel of 4-factor operator $\Psi_{4}(h)$

$$
\begin{aligned}
\operatorname{Ker}^{4} \Psi_{4}(h)= & \left\{h=\left(h_{x}, h_{\mu}, h_{\alpha}\right) \in C^{2}[0,2 \pi] \times \mathbb{R} \times \mathbb{R}:\right. \\
& \left.\frac{d^{2} h_{x}}{d t^{2}}+h_{x}+\frac{24}{\pi} \sin t \int_{0}^{2 \pi}\left(h_{x}^{3} h_{\mu}-\sin \tau h_{\alpha}^{4}\right) \sin \tau d \tau=0\right\} .
\end{aligned}
$$


Taking into account the equations

$$
\int_{0}^{2 \pi} \sin ^{4} \tau d \tau=\frac{3}{4} \pi, \quad \int_{0}^{2 \pi} \sin ^{2} \tau d \tau=\pi
$$

and the fact that $h_{x}=z \sin \tau$, since $h_{x} \in \operatorname{Ker} \bar{F}_{x}^{\prime}(0,0,0)$, we solve the following equation of unknowns $z, h_{\mu}, h_{\alpha}$ :

$$
\frac{24}{\pi} \sin t\left[z^{3} h_{\mu} \int_{0}^{2 \pi} \sin ^{4} \tau d \tau-h_{\alpha}^{4} \int_{0}^{2 \pi} \sin ^{2} \tau d \tau\right]=0 .
$$

From here $\frac{3}{4} z^{3} h_{\mu}-h_{\alpha}^{4}=0$ and $z=\sqrt[3]{\frac{4 h_{\alpha}^{4}}{3 h_{\mu}}}$ for $h_{\mu} \neq 0$. We assume here that $\|h\|=\|\left(h_{x}, h_{\mu}\right.$, $\left.h_{\alpha}\right) \|=1$ (the vector $h$ lies on a ball of radius 1 ), where we define the norm $\|\cdot\|$ in the space $\mathcal{C}^{2}[0,2 \pi]$ as follows:

$$
\forall x \in \mathcal{C}^{2}[0,2 \pi] \quad\|x\|=\sup _{t \in[0,2 \pi]}|x(t)|+\sup _{t \in[0,2 \pi]}\left|x^{\prime}(t)\right|+\sup _{t \in[0,2 \pi]}\left|x^{\prime \prime}(t)\right|,
$$

while the norm in the space $\mathbb{R} \times \mathbb{R}$ as $\|(y, z)\|=|y|+|z|$. We can accept the norm in the space $\mathcal{C}^{2}[0,2 \pi] \times \mathbb{R} \times \mathbb{R}$ as standard, i.e.,

$$
\|(x, y, z)\|=\|x\|_{\mathcal{C}^{2}[0,2 \pi]}+\|(y, z)\|_{\mathbb{R} \times \mathbb{R}} .
$$

Then we describe the space $\operatorname{Ker}^{4} \Psi_{4}(h)$ clearly as follows:

$$
\operatorname{Ker}^{4} \Psi_{4}(h)=\left\{\left(\sqrt[3]{\frac{4 \alpha^{4}}{3 \mu}} \sin t, \mu, \alpha\right)\right\} \cup\{(c \sin t, 0,0)\} \cup\{(0, \mu, 0)\},\left\|\left(h_{x}, \mu, \alpha\right)\right\|=1,
$$

where $h_{x}$ equals $\sqrt[3]{\frac{4 \alpha^{4}}{3 \mu}} \sin t, c \sin t$ for $c \in \mathbb{R}$ or 0 .

At the end we will examine the surjectivity of 4-factor operator on the 4-kernel. Note that vectors $(c \sin t, 0,0)$ and $(0, \mu, 0)$ are solutions of the Duffing equation, then we are interested in vectors of the form $H=\left(\sqrt[3]{\frac{4 \alpha^{4}}{3 \mu}} \sin t, \mu, \alpha\right)$, where $3 \sqrt[3]{\frac{4 \alpha^{4}}{3 \mu}}+|\mu|+|\alpha|=1$. So we will verify whether

$$
\forall y \in \mathcal{C}[0,2 \pi] \exists u=\left[h_{u}, h_{\lambda}, h_{\beta}\right] \in \mathcal{C}^{2}[0,2 \pi] \times \mathbb{R} \times \mathbb{R} \quad \Psi_{4}(H)[u]=y .
$$

We have

$$
\begin{aligned}
\Psi_{4}(H)[u]= & \frac{d^{2} h_{u}}{d t^{2}}+h_{u}+\frac{1}{\pi} \sin t \int_{0}^{2 \pi}\left(18\left(\sqrt[3]{\frac{4 \alpha^{4}}{3 \mu}}\right)^{2} \sin ^{2} \tau \mu h_{u}\right. \\
& \left.+8 \frac{\alpha^{4}}{\mu} \sin ^{3} \tau h_{\lambda}-24 \sin \tau \alpha^{3} h_{\beta}\right) \sin \tau d \tau \\
= & y .
\end{aligned}
$$

Using now Lemma 10, p.6 from [8], i.e., putting $y=y_{4}=a \sin t \in Y_{4}$, we find the element $h_{u}=b \sin t \in \operatorname{Ker} \bar{F}_{x}^{\prime}(0,0,0)$. Namely, we obtain

$$
\frac{1}{\pi} \sin t \int_{0}^{2 \pi}\left(18\left(\sqrt[3]{\frac{4 \alpha^{4}}{3 \mu}}\right)^{2} \mu b \sin ^{4} \tau+8 \frac{\alpha^{4}}{\mu} \sin ^{4} \tau h_{\lambda}-24 \alpha^{3} h_{\beta} \sin ^{2} \tau\right) d \tau=a \sin t
$$


This implies $b=\frac{1}{9 \sqrt[3]{6 \alpha^{8} \mu}}\left(a+24 \alpha^{3} h_{\beta}-\frac{6 \alpha^{4}}{\mu} h_{\lambda}\right)$ and

$$
h_{u}=\frac{1}{9 \sqrt[3]{6 \alpha^{8} \mu}}\left(a+24 \alpha^{3} h_{\beta}-\frac{6 \alpha^{4}}{\mu} h_{\lambda}\right) \sin t .
$$

The solutions of the above equation exist, hence 4-factor operator is a surjection on the element $H$.

Summing up, we conclude from the generalized Lyusternic theorem: for $x^{*}=(0,0,0)$, the tangent cone $T_{x *} M$ to the solutions set $M$ coincides with the kernel of the fourth derivative of the mapping $\Psi_{4}$, i.e., with the set $H_{4}\left(x^{*}\right)=\operatorname{Ker}^{4} \Psi_{4}(H)$. Thus there exist nontrivial solutions of the Duffing equation (1), and we can write them in the form $x(\varepsilon, t)=x^{*}+\varepsilon H+r(\varepsilon)$, with $\|r(\varepsilon)\|=o(\varepsilon)$, for $\varepsilon \in(0, \delta)$, where $\delta>0$ is sufficiently small. Let $\bar{\beta}, \bar{\mu}$ be fixed numbers such that the equation $3 \sqrt[3]{\frac{4 \bar{\beta}}{3 \bar{\mu}}}+|\bar{\mu}|+\left|\bar{\beta}^{\frac{1}{4}}\right|=1$ holds, where $\bar{\mu}=\bar{\mu}\left(\bar{\beta}^{\frac{1}{4}}\right), \bar{\beta}=\bar{\alpha}^{4}$ and $0<\bar{\beta}^{\frac{1}{4}}<1$. Putting $x^{*}=(0,0,0)$, and $\varepsilon=\frac{\beta^{\frac{1}{4}}}{\bar{\beta}^{\frac{1}{4}}}$, we get formulas (14), (15), and this finishes the proof of the theorem.

\section{Conclusion}

In this paper we used $p$-regularity theory to prove the existence of solutions of the nonlinear Duffing equation and gave an approximate description of the solutions set. Compared with the latter paper by Grzegorczyk et al. [1], where only 2-regularity was studied, the results of the present paper allow one to study the regularity of higher order (4-regularity) and apply it for seeking approximative solutions of nonlinear boundary value problems. We showed that the method of $p$-regularity may have an effective application to the study of solutions of advanced differential equations. This construction may be generalized for investigation of the case $p>4$.

\section{Acknowledgements}

This work was supported by the Russian Foundation for Basic Research Grant No.14-07-00805, by the Leading Research Schools Grant No.NSH-8860.2016.1 and by the Russian Academy of Sciences Presidium Program I-33 P RAS.

\section{Competing interests}

The authors declare that they have no competing interests.

\section{Authors' contributions}

All authors read and approved the final manuscript.

\section{Author details}

${ }^{1}$ Siedlce University of Natural Sciences and Humanities, 3-go Maja 54, Siedlce, 08-110, Poland. ${ }^{2}$ System Research Institute, Polish Academy of Sciences, Newelska 6, Warsaw, 01-447, Poland. ${ }^{3}$ Dorodnicyn Computer Center, Russian Academy of Sciences, Vavilova 40, Moscow, 119991, Russia.

\section{Publisher's Note}

Springer Nature remains neutral with regard to jurisdictional claims in published maps and institutional affiliations.

Received: 21 February 2017 Accepted: 24 May 2017 Published online: 06 June 2017

\section{References}

1. Grzegorczyk, W, Medak, B, Tret'yakov, AA: Application of $p$-regularity theory to nonlinear boundary value problems. Bound. Value Probl. 2013, 251 (2013). http://www.boundaryvalueproblems.com/content/2013/1/251

2. Izmailov, AF, Tret'yakov, AA: Factor-Analysis of Nonlinear Mappings. Nauka, Moscow (1994) (in Russian)

3. Izmailov, AF, Tret'yakov, AA: 2-Regular Solutions of Nonlinear Problems. Theory and Numerical Methods. Nauka, Moscow (1999) (in Russian)

4. Tret'yakov, AA: The implicit function theorem in degenerate problems. Russ. Math. Surv. 42, 179-180 (1987)

5. Tret'yakov, AA, Marsden, JE: Factor analysis of nonlinear mappings: p-regularity theory. Commun. Pure Appl. Anal. 2(4), 425-445 (2003) 
6. Bender, CM, Orszag, SA: Advanced Mathematical Methods for Scientists and Engineers I: Asymptotic Methods and Perturbation Theory, pp. 545-551. Springer, Berlin (1999)

7. Tajaddodianfar, F, Yazdi, MRH, Pishkenari, HN: Nonlinear dynamics of MEMS/NEMS resonators: analytical solution by the homotopy analysis method. Microsyst. Technol. 23(6), 1913-1926 (2017). doi:10.1007/s00542-016-2947-7

8. Medak, B, Tret'yakov, AA: Existence of periodic solutions to nonlinear $p$-regular boundary value problem. Bound. Value Probl. 2015, 91 (2015). doi:10.1186/s13661-015-0360-2

9. Medak, B: Development of p-regularity apparatus and its application to describing the structure of solution sets of degenerated differential equations. Doctoral thesis, UMCS, Lublin (2013). (in Polish)

Submit your manuscript to a SpringerOpen ${ }^{\odot}$ journal and benefit from:

- Convenient online submission

- Rigorous peer review

- Immediate publication on acceptance

- Open access: articles freely available online

- High visibility within the field

- Retaining the copyright to your article 